\title{
Asymmetry of Left Versus Right Lateral Face in Face Recognition*
}

\author{
Wenfeng Chen ${ }^{1}$, Chang Hong $\mathrm{Liu}^{2}$, and Xiaolan $\mathrm{Fu}^{1}$ \\ ${ }^{1}$ State Key Laboratory of Brain and Cognitive Science, \\ Institute of Psychology, Chinese Academy of Sciences, Beijing 100101, China \\ \{chenwf, fuxl\}@psych.ac.cn \\ ${ }^{2}$ Department of Psychology, University of Hull, Cottingham Road, Hull, HU6 7RX, UK \\ c.h.liu@hull.ac.uk
}

\section{Introduction}

Prior research has found that the left side of the face is emotionally more expressive than the right side [1]. This was demonstrated in a study where the right and the left halves of a face image were combined with their mirror-reversed duplicates to make composite images. When observers were asked which composite face appeared more emotional, they selected the left-left over the right-right composite more often.

According to Nicholls et al., this asymmetrical display of facial expression may be a part of an innate code for facial expressions [1]. The identity of the face, on the other hand, requires learning or a process of familiarization. There has been evidence that recognition facial identify can be influenced by recognition of facial expressions [2]. The present research attempts to resolve an unanswered question in this literature: can the hardwired asymmetry in facial expression affect the learning of facial identities? Faces in reality are often seen from the left or right views. Because recognition of novel faces is viewpoint dependent, we ask whether the face can be recognized more easily from the left or right views.

\section{Methods}

Twenty two undergraduates participated in the experiment. A total of 84 faces were chosen from a 3D face database [3]. Nine of these were used a practice session. The models were rendered and converted to 2D grayscale images with black background. Each face had 3 different poses (frontal $/ 0^{\circ}, 30^{\circ}$ to the left, and $30^{\circ}$ to the right), and 7 facial expressions (happiness, sadness, disgust, surprise, anger, fear, and neutral).

Each trial of the experiment consisted of two sequentially displayed faces, a learn face and a test face. Participants were asked to judge whether the two faces are of the same person. They were told to respond as quickly and as accurately as possible. The learn face was either shown in six frontal images of different facial expressions that appeared successively (multiple-image condition), or a single frontal image with an emotional or neutral expression (one-image conditions). The face in all these

\footnotetext{
* This research was supported in part by grants from 973 Program (2006CB303101), the NSFC (60433030, 30500157, and 30600182) and China-UK Science Networks.
} 
conditions was shown for $3 s$. The test face was always shown in a neutral expression, and was presented in a different view and size from the learned face. Half of the test faces faced left, and the remaining half faced right.

\section{Results and Discussion}

Face recognition performance based on the right side of the face was superior to the left side (see the accuracy results in figure 1). However, this asymmetry in face recognition demonstrates a right-face advantage, which is the opposite of the left-face advantage for facial expressions [1].
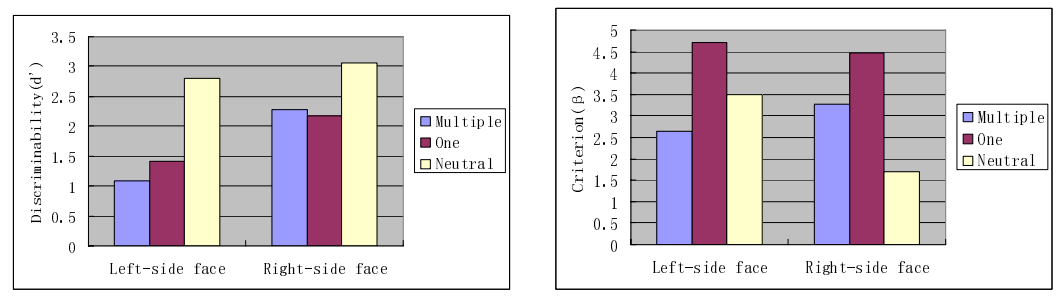

Fig. 1. Means of accuracy $\left(d^{\prime}\right)$ and criterion $(\beta)$ data in the face matching task

Our finding may result from a side effect of the left-face advantage for facial expressions. Because the stronger expression from the left side of the face is attributable to a greater difference between the emotional and neural expressions on this side, the larger physical difference between learned and tested faces should make this side of the face more difficult to recognize. If this is true, the asymmetry should vanish in the neutral expression condition where no expression change occurred. Our analysis of data support this explanation: the asymmetry effect appeared only when expressions changed between learning and test. However, McCurdy observed that the right side of the face is noticeably more like the whole face than the left side [4]. This may be an alternative explanation that focuses on a possible, inherent advantage of the right side for face identity. More rigorous design is needed to test these theories. For example, using left/right composite faces in future studies should allow precise estimation of the contribution from the two halves of the face in recognition tasks.

\section{References}

1. Nicholls, M.E.R., Wolfgang, B.J., Clode, D., Li, A.K.: The effect of left and right poses on the expression of facial emotion. Neuropsychologia. 40, 1662-1665 (2002)

2. Ganel, T., Goshen-Gottstein, Y.: Effects of Familiarity on the Perceptual Integrality of the Identity and Expression of Faces: The Parallel-Route Hypothesis Revisited. Journal of Experimental Psychology: Human Perception and Performance 30, 583-597 (2004)

3. Yin, L., Wei, X., Sun, Y., Wang, J., Rosato, M.J.: A 3D facial expression database for facial behavior research. 7th International Conference on Automatic Face and Gesture Recognition (FGR06), pp. 211-216.

4. McCurdy, H.G.: Experimental notes on the asymmetry of the human face. Journal of Abnormal Psychology. 44, 553-555 (1949) 